\title{
Individual response to antidepressants for depression in adults - a simulation study and meta-analysis
}

\author{
Klaus Munkholm, M.D., DMSc. ${ }^{1}$, Stephanie Winkelbeiner, Ph.D. ${ }^{2}$, \& Philipp Homan, M.D., Ph.D. ${ }^{2}$ \\ ${ }^{1}$ Nordic Cochrane Centre, Rigshospitalet, Copenhagen, Denmark \\ ${ }^{2}$ University Hospital of Psychiatry Zurich, University of Zurich, Zurich, Switzerland
}

\begin{abstract}
The observation that some patients appear to respond better to antidepressants for depression than others encourages the assumption that the effect of antidepressants differs between individuals and that treatment can be personalized. To test this assumption, we compared the outcome variance in the group of patients receiving antidepressants with the outcome variance of the group of patients receiving placebo in randomized controlled trials (RCTs) of adults with major depressive disorder (MDD). An increased variance in the antidepressant group would indicate individual differences in response to antidepressants. In addition, we illustrate in a simulation study why attempts to personalize antidepressant treatment using RCTs might be misguided. We first illustrated the variance components of trials by simulating RCTs and crossover trials of antidepressants versus placebo. Second, we analyzed data of a large meta-analysis of antidepressants for depression, including a total of 222 placebo-controlled studies from the dataset that reported outcomes on the 17 or 21 item Hamilton Depression Rating Scale or the Montgomery-Åsberg Depression Rating Scale. We performed inverse variance, random-effects meta-analyses of the variability ratio (VR) between the antidepressant and placebo groups. The meta-analyses of the VR comprised 345 comparisons of 19 different antidepressants with placebo in a total of 61144 adults with an MDD diagnosis. Across all comparisons, we found no evidence for a larger variance in the antidepressant group compared with placebo overall $\left(\mathrm{VR}=1.00,95 \% \mathrm{CI}: 0.98 ; 1.01, \mathrm{I}^{2}=0 \%\right)$ or for any individual antidepressant. Our findings did not provide empirical support for individual differences in response to antidepressants.
\end{abstract}

Keywords: antidepressants, depression, individual treatment response, variability ratio, variance components.

\section{Introduction}

Major depressive disorder (MDD) is considered a common ${ }_{15}$ illness [1] and by the World Health Organization estimated to be the leading cause of disability worldwide [2]. Antide- ${ }_{17}$ pressants, either alone or in combination with psychotherapy, ${ }_{18}$ are recommended by guidelines for the treatment of MDD 19 [3-7]. Yet, the effect of antidepressants on depression symp- ${ }_{20}$ toms is small compared with placebo [9-16]; the difference ${ }_{21}$ in depression symptom severity reduction due to antidepres- 22 sants compared with placebo in short-term studies is approx- ${ }_{23}$ imately 2 points $[13,17]$ on the 17 -item Hamilton Depression ${ }_{24}$

Correspondence concerning this article should be addressed ${ }^{28}$ to Klaus Munkholm, M.D., DMSc., Nordic Cochrane Centre, ${ }^{29}$ Rigshospitalet, Dept. 7811, Blegdamsvej 9, DK-2100 Copenhagen, 30 Denmark. E-mail: km@cochrane.dk
2 Rating Scale (HAMD) [18] (range 0-52). However, considering the methodological limitations of many antidepressant trials [19] , the true effect of antidepressants for depression in adults remains uncertain [17].

The small apparent effect of antidepressants over placebo [10] is often believed to result from some patients having substantial benefit, while others have less or no benefit of the treatment $[1,20,21]$. Thus, a common interpretation of the observed heterogeneity in outcomes among patients treated with antidepressants, whether in clinical practice or in the context of a randomized controlled trial (RCT), is that those with the best outcome, often labeled "responders", differ from those with less favorable outcomes, similarly labeled "non-responders". The implicit assumption is that response is a permanent characteristic of the individual patient, and the observed variability in outcomes can be ascribed to heterogeneity in the response to antidepressants. This perceived heterogeneity has motivated efforts to direct the research and treatment agenda into one of personalized medicine. The aim 
is to match the individual patient to a therapy that best suits 84 their specific characteristics and condition [22]. Personalized medicine includes the search for potential pharmacogenetic ${ }_{85}$ markers [23,24], other biomarkers [25,26], and clinical characteristics [27]. Those are hoped to predict the response to antidepressants and ultimately help to identify the patients ${ }^{86}$ that are most likely to "respond" to antidepressants. Without ${ }^{87}$ such prediction markers, the individual patient is subject to ${ }^{88}$ a trial-and-error process where different antidepressants may ${ }^{89}$ be given in succession until the desired outcome is reached ${ }^{90}$ $[28,29]$.

There may, however, be little reason to assume that a drug ${ }^{92}$ that appears to be marginally effective in a larger population, ${ }^{93}$ would turn out to be more effective in a subpopulation [30], ${ }^{94}$ let alone in certain individuals. The promise of personalized ${ }^{95}$ antidepressant treatment may thus rest largely on untested ${ }^{96}$ assumptions about individual differences in response to the ${ }^{97}$ treatment.

Estimating individual response to treatment, known as the ${ }^{99}$ treatment-by-patient interaction, is complex and cannot be $\mathrm{e}^{100}$ inferred from an RCT. The design of an RCT allows simply ${ }^{101}$ to compare the treatment with the control group and with ${ }^{102}$ that to estimate an average effect of the treatment. While $i^{103}$ is common to uncritically attribute the variation in outcomes ${ }^{104}$ observed among patients in RCTs to characteristics of the ${ }^{105}$ individual, the more likely interpretation that these differ- ${ }^{106}$ ences in response reflect random variation is often not con- ${ }^{107}$ sidered [31]. RCTs do not allow to distinguish between in- ${ }^{108}$ dividual responses to the treatment and random variability or ${ }^{109}$ any scenario in between [31]. To determine on an individual ${ }^{110}$ level whether a drug works thus depends on the comparison within patients to phases without the treatment - a counterfactual[30]; therefore, designs such as repeated crossover tri- ${ }^{111}$ als are required [32].

While RCTs cannot directly distinguish individual response ${ }^{112}$ to treatment from other components of variation, they may ${ }^{113}$ provide indirect evidence about the presence of individual ${ }^{114}$ differences in response. This indirect evidence is comprised ${ }^{115}$ by the variance of the treatment compared to the control ${ }^{116}$ group [32]. An increased variance in the treatment group ${ }^{117}$ compared to the control group could indicate the presence of ${ }^{118}$ individual differences in response to antidepressants, known ${ }^{119}$ as the treatment-by-patient interaction [32-34]. Follow-120 ing this rationale, we recently showed that evidence of $\mathrm{a}^{121}$ treatment-by-patient interaction in RCTs of antipsychotics ${ }^{122}$ compared with placebo for schizophrenia was surprisingly ${ }^{123}$ small[35]. Here, we extended this work to antidepressants. ${ }^{124}$ We investigated whether there is empirical evidence for $a^{125}$ individual difference in response to antidepressants in RCTs. ${ }^{126}$ Additionally, we illustrated the different components of vari-127 ation in RCTs and crossover trials using simulated data to ${ }^{128}$ highlight the component of interest: the treatment-by-patient ${ }^{129}$ interaction.

\section{Methods}

\section{Trial simulation}

To illustrate the different components of variation, we simulated an RCT with 30 adults with MDD, informing the parameters by a published RCT of sertraline versus placebo [36]. Accordingly, patients were randomized to either treatment with the antidepressant sertraline or placebo. Symptom severity was assessed with the 17-item HAMD with a mean baseline score of 22 points and a mean (standard deviation (SD)) baseline-to-endpoint change score of 13 (6) points in the treatment and 11 (6) points in the placebo groups, respectively.

We first simulated the data to demonstrate the variation in effects across both groups and the variation between patients in the treatment group. We illustrated the effect of dichotomization of the treatment group into categories of "responders" and "non-responders", depending on whether the patients' endpoint score decreased by $50 \%$ or more compared with baseline [37]. Second, we simulated a crossover trial by adding a placebo condition for the patients in the treatment group to show the consequence of between-patient variation. Third, we simulated the repeated measurement over time to explain the component of random within-patient fluctuation of symptoms. Lastly, we simulated a double crossover trial, to elucidate how such a design allows to separate the treatment-by-patient interaction from other variance components.

\section{Meta-analysis}

We used the data of a recent meta-analysis of antidepressants for depression in adults, comprising 522 studies and 116477 patients [10]. The authors of the meta-analysis had searched the Cochrane Central Register of Controlled Trials, CINAHL, Embase, LILACS database, MEDLINE, MEDLINE In-Process, PsycINFO, AMED, the UK National Research Register, and PSYNDEX from the date of their inception to January 8,2016 . The search was performed with no language restrictions, supplemented with manual searches for published, unpublished, and ongoing RCTs in international trial registers, websites of drug approval agencies, and key scientific journals in the field [10]. Included were double-blinded RCTs comparing antidepressants (provided dosing was within the therapeutic range) with placebo or another antidepressant as oral monotherapy for the acute treatment of adults ( $\geq 18$ years of age, both sexes) with a primary MDD diagnosis [10]. Further, the included antidepressants were second-generation antidepressants approved by the regulatory agencies in the USA, Europe, or Japan, the tricyclics amitriptyline and clomipramine included in the World Health 
Organization Model List of Essential Medicines, and tra-177 zodone and nefazodone, which were included because of 178 their "distinct effect and tolerability profiles" [10]. This data179 is available online, accompanying the published article [10] $]_{180}$ (https://data.mendeley.com/datasets/83rthbp8ys/2).

Eligibility criteria. We applied additional eligibility criteria to this dataset [10] including only placebo-controlled studies that reported the sample size, mean and SD of baseline-to-endpoint change scores or raw endpoint scores on either the HAMD-17, HAMD-21 [18], or the ${ }_{182}$ Montgomery-Åsberg Depression Rating Scale (MADRS) ${ }_{183}$ [38], which were the most frequently used scales among the ${ }_{184}$ studies [17].

Study records, selection process, and data collec-186 tion. We downloaded the full online dataset (https://data. ${ }^{187}$ mendeley.com/datasets/83rthbp8ys/2) and imported it into ${ }^{188}$ the statistical software R (version 3.6.0). We selected studies ${ }^{189}$ based on the information in the online dataset and refrained 190 from collecting additional data. From the dataset, we ex-191 tracted information on study identification (e.g. first author, ${ }^{192}$ trial registration number), study year, mean (SD) raw end-193 point or baseline-to-endpoint change score on the HAMD-194 17, HAMD-21, and MADRS, and sample size.

195 Statistical analysis. The SDs of the baseline-to-endpoint ${ }^{196}$ change scores or raw endpoint scores of the antidepressant ${ }^{197}$ and placebo group include the same variance components. ${ }^{190}$ The antidepressant group may, however, in addition contains ${ }^{199}$ a possible treatment-by-patient interaction, indicating indi- ${ }^{200}$ vidual response differences. A higher variance in the antide-201 pressant group compared with placebo thus would indicate 202 the presence of a treatment-by-patient interaction. To test $t_{203}$ this hypothesis, we calculated the relative variability of the 204 antidepressant and placebo group for each study as the $\log _{205}$ variability ratio (log VR) [39] with

$$
\log V R=\log \left(\frac{S D_{t x}}{S D_{c t}}\right)+\frac{1}{2\left(N_{t x}-1\right)}-\frac{1}{2\left(N_{c t}-1\right)}
$$

where SD is the reported sample SD for the treatment (tx) and the control (ct) group, and $\mathrm{N}$ the respective sample size ${ }_{208}$ (Nakagawa et al., 2015). We further calculated the corre- ${ }_{209}$ sponding sampling variance, $\mathrm{SD}^{2}{ }_{\mathrm{logVR}}$, for each comparison ${ }_{210}$ of an antidepressant with placebo with

$$
S D_{\log V R}^{2}=\frac{1}{2\left(N_{t x}-1\right)}+\frac{1}{2\left(N_{c t}-1\right)}
$$

The log VR may be limited in its applicability in situations ${ }^{215}$ of a dependency of the mean and variance (Nakagawa et al., ${ }^{216}$ 2015). Therefore, we checked for a mean-variance relation- ${ }^{217}$ ship by fitting a linear model with the SDs of the baseline-toendpoint change scores or raw endpoint scores as the depen-219 dent variable, the mean of the baseline-to-endpoint change 220 scores or raw endpoint scores as the predictor and weighted this by the sample size. In case of a mean-variance relationship, we calculated the log coefficient of variation ratio (log CVR) as an additional effect size statistic (Nakagawa et al., 2015) with

$$
\log C V R=\log \left(\frac{C V_{t x}}{C V_{c t}}\right)+\frac{1}{2\left(N_{t x}-1\right)}-\frac{1}{2\left(N_{c t}-1\right)}
$$

where CVtx and CVct are SDtx $/ \bar{x}$ tx and SDct $/ \bar{x}$ ct, respectively. We weighted the $\log$ VR and $\log$ CVR with the inverse of their corresponding sampling variance [40] and entered them into a random-effects model using restricted maximum-likelihood estimation. We back-transformed the results to obtain a VR that indicates greater variability in the antidepressant group compared with placebo for values greater than 1 and less variability in the antidepressant group compared with placebo for values smaller than 1 . For studies that investigated multiple antidepressants or doses compared to placebo, we divided the sample size of the placebo group by the number of treatment arms while retaining the mean and SD and creating multiple pair-wise comparisons for those studies. Our primary outcome was the overall summary estimate for the VR across all included comparisons. Further, we conducted different subgroup analyses (1) by type of antidepressant, (2) by symptom severity scale, (3) and by the fact of whether outcomes were reported as raw endpoint scores or baseline-to-endpoint change scores.

Data and code availability. All analyses were performed with the R packages metafor, version 2.1.0 [40], and meta, version 4.9.6 [41]. All data and code are available online (https://osf.io/5gpe4/). This study was pre-registered on the Open Science Framework platform (https://osf.io/cy2rt).

\section{Results}

\section{Simulation study}

Plotting the baseline-to-endpoint change scores for both the treatment and control group of an RCT, we may observe differences in response to the antidepressant (Figure 1a). Ignoring the variation in outcomes in the control condition and focusing on the variation between individuals in the treatment condition only, we may rank patients according to their outcome and infer that some patients responded better to the treatment than others (Figure 1b). This perception may be strengthened when further dichotomizing the patients in the treatment group as either "responders" or "non-responders" (Figure 1c).

Adding a simulated crossover condition to the initial RCT (Figure 1a) allows the within-patient comparison between 


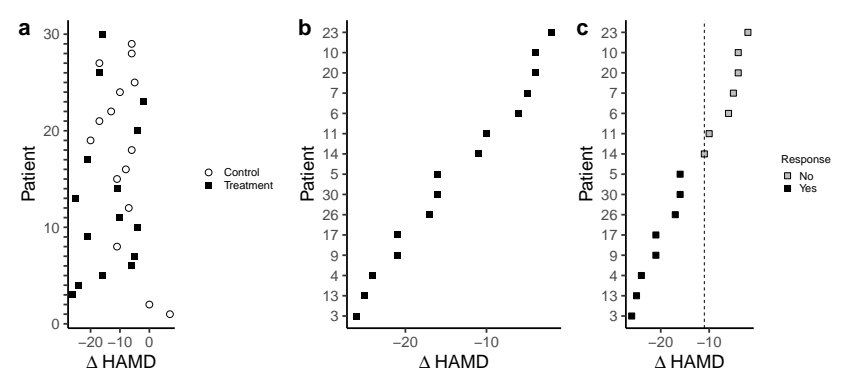

Figure 1. Simulation of an RCT with 30 patients with MDD randomized to antidepressants or placebo with a mean difference between groups of 2 points on the HAMD17. Plotting the observed outcomes of both the antidepressant and placebo group shows variation and overlap in the outcomes in the two groups (a). Observing only the variation in the outcomes in the antidepressant group, while ignoring the variation in the placebo group, and ranking patients according to their outcome can encourage the assumption that the effect differs between individuals (b). Dichotomizing the outcomes based on arbitrary thresholds and the subsequent categorizing of patients as "responders" and "non-responders" can accentuate the perception of individual differences in treatment response and tempt into interpreting those as stable characteristics of the individual patient (c).

the antidepressant and the placebo condition. Only by comparing the response to the antidepressant to the response to placebo within a patient is a comparison of the effect of the treatment, rather than just the observed outcome, between patients possible. Inferences about whether the antidepressant effect is constant across patients (Figures $2 \mathrm{a}$ and $2 \mathrm{~b}$ ) or whether the effect of antidepressants is different in each patient (Figures $2 \mathrm{c}$ and $2 \mathrm{~d}$ ) are now possible.

In addition to the between-patient variation, RCTs include yet another component of variation: the within-patient variation. Symptoms may fluctuate randomly over time within a patient (Figure 3a). While a larger variation may be observed in some patients compared with others, all might have had the same mean symptom severity when averaging observations over time (Figure $3 b$ ). It is thus possible that the within-patient variation alone can explain the variation in the observed outcome in an RCT as well as the variation in the net-benefit observed in a single crossover trial.

While a simple crossover trial accounts for between-patient variation in effects, it does not provide information on whether the observed effect in a given patient is a constant feature of that individual. This is only possible by repeating the crossover trial (Supplementary Figure 1) [32,35]. In a repeated crossover study, equal variances would be observed in case of a constant treatment effect across all patients; a consequence of a constant effect is that the treatment does
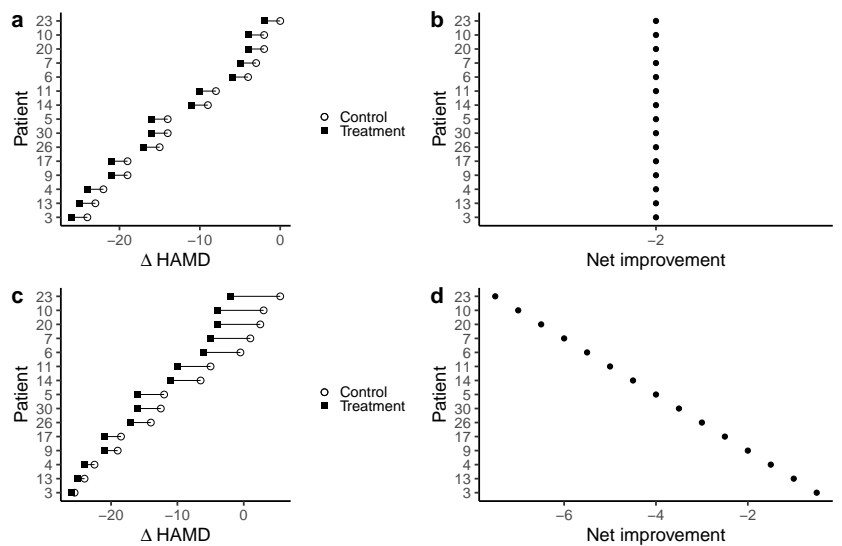

Figure 2. Simulation of adding a single crossover condition to the initial RCT shows that inferences about individual treatment effects can be misleading when only considering the observed outcomes. When adding a crossover condition, it is possible that the outcomes observed in the patients under the antidepressant condition would be paralleled by the outcomes under a crossover placebo condition (a). In such a scenario, the net benefit, the actual effect of the treatment, is constant between individuals (b). Patients classified as "responders" when just observing their outcome under the treatment condition (Figure 1c) would thus not differ from those classified as "non-responders". It is also possible, that the patients with the best outcome under the initial treatment condition would also have the best outcomes in the crossover control condition (c). In such a scenario, the patients with the best outcome would, in fact, experience the smallest net benefit of the treatment (d). Those patients that would otherwise be classified as "non-responders" based on their observed outcomes (Figure 1c) would experience the largest net benefit.
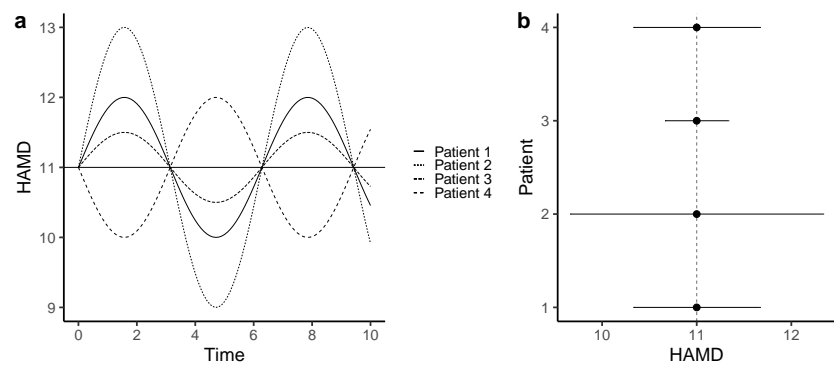

Figure 3. Simulation of repeated measurements of depression symptom severity on the HAMD17 among 4 patients of the initial RCT simulation (Figure 1a). The HAMD17 score may fluctuate over time independently of the intervention (a). While the mean HAMD17 score may be the same across the patients, the amount of variation may differ (b). In such a scenario, within-patient variation may account for the entire variation in the observed outcome in an RCT. 


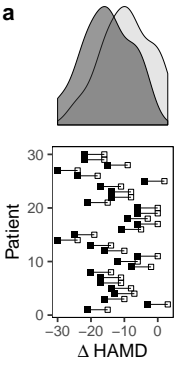

a Control - Treatme

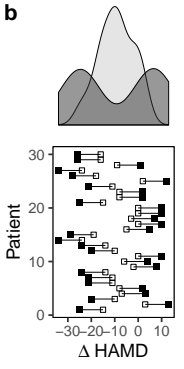

- Control - Treatmen

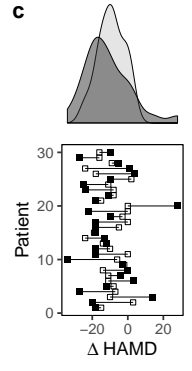

Control - Treatme

Figure 4. Simulation of a crossover trial of 30 patients with MDD randomized to subsequently receive an antidepressant and placebo with a mean improvement of 2 points on the HAMD17. The marginal density plots illustrate the distribution of outcomes in the two conditions in three different scenarios: (a) illustrates a scenario with a constant treatment effect, in which the variances in the two groups would be equal; (b) shows a scenario with two subpopulations with different effects. Although the effect is the same for all patients in each subgroup, the distribution in the treatment condition has higher variability than the placebo condition; (c) shows a scenario with a variable effect in each patient in which greater variability is observed in the treatment condition.

not affect variability (Figure 4a). An increased variance in the treatment condition relative to the control condition may arise in one of two different scenarios. One scenario is the existence of two subpopulations with different responses to the antidepressant, a treatment-by-subgroup interaction (Figure $4 b)$. Such a scenario would call for stratified medicine, in which treatment with an antidepressant is conditional on the patient belonging to a subgroup of individuals that share certain characteristics. An alternative scenario resulting in increased variance in the observed effect in the treatment condition compared with the control condition is the existence of a variable effect in each individual, a treatment-by-patient interaction, without any subgroup sharing a common effect272 (Figure 4c). Such a scenario would call for personalized273 medicine in which the antidepressant would be conditional274 on features unique to the individual.

How differences in the variances of the antidepressant and ${ }_{277}^{276}$ placebo group will be reflected by the VR depends on the distribution of the individual response to antidepressant and the magnitude of the response. The VR will be higher in situations where many individuals have large effects and lower in situations where individuals have small effects (Figure 5) ${ }_{282}$

\section{Meta-analysis of empirical data}

We included 345 comparisons from 222 RCTs that investi- ${ }^{286}$ gated a total of 19 antidepressants. The studies included a287

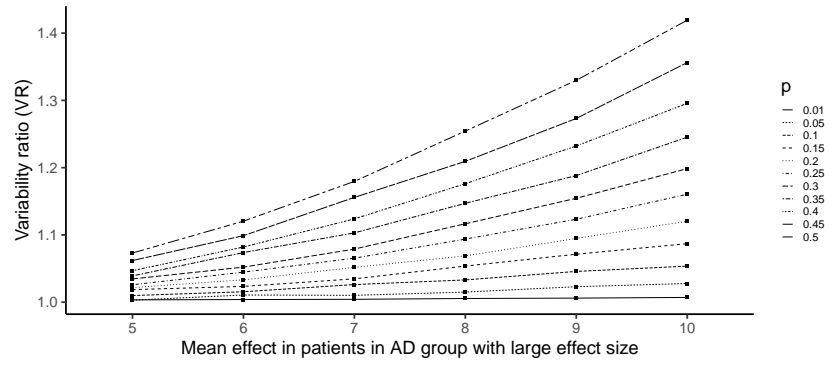

Figure 5. Simulation of 1000 trials of antidepressants versus placebo with 300 patients in each group, with an average effect of 2 (6) points on the HAMD17. We simulated multiple scenarios with increasing effects of antidepressants in increasing proportions of the patients randomized to antidepressants. We assumed a normal distribution for the effects, but the true distribution, given individual responses, could have other shapes.

p: proportion of patients in the antidepressant group with a larger effect of antidepressants than the average 2 points on the HAMD17; AD: antidepressant.

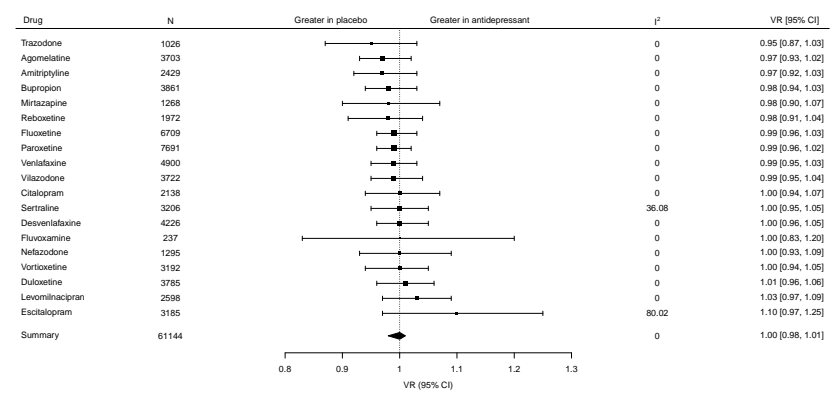

Figure 6. Forest plot of the VR for each antidepressant versus placebo.

VR: Variability ratio; $\mathrm{I}^{2}$ : inconsistency (\%); CI: confidence interval.

total of 61144 patients of which 38254 patients received an antidepressant and 22890 received placebo. Details of the included studies are available in the Supplementary Tables 1 and 2. Across all comparisons, we found no difference in variance of the antidepressant and placebo group $(V R=1$, $95 \%$ CI: $0.98 ; 1.01, \mathrm{I}^{2}=0 \%$ ). In the subgroup analysis by antidepressant, there was no difference in variance between any antidepressant and placebo (Figure 6) and no difference in VR between drugs ( $\mathrm{df}=18, P=0.98$ ). The inconsistency observed for studies of escitalopram $\left(\mathrm{I}^{2}=80 \%\right)$, which could indicate between-study heterogeneity, appeared to be due to a single study [42] with a very large effect. After excluding this study [42] no inconsistency was observed in the meta-analysis of escitalopram compared with placebo (Supplementary Figure 2).

In the subgroup analysis by rating scale, we found no differ- 
ence in variance of the antidepressant and placebo group for 338 any of the studies reporting on the HAMD-17, HAMD-21 or 339 MADRS, respectively, with no difference in VR between the 340 groups (df $=2, P=0.86$; Supplementary Table 3). In the 341 subgroup analysis by outcome measure, we found no differ-342 ence in the variance of the antidepressant and placebo group ${ }_{343}$ for either studies using the raw endpoint scores or for studies344 using baseline-to-endpoint change as the outcome, with no ${ }_{345}$ difference between the two groups of studies ( $\mathrm{df}=1, P=346$ 0.07; Supplementary Table 4)

Linear models indicated a statistically significant mean ${ }^{348}$ variance relationship (Supplementary Figure 2). Therefore, we conducted an additional random-effects meta-analysis with the $\log$ CVR as the effect size statistic. In this model, the CVR was lower in the antidepressant group compared with placebo $\left(C V R=0.91,95 \%\right.$ CI: $\left.0.89,0.93, \mathrm{I}^{2}=46.46 \%\right)$.

\section{Discussion}

This study investigated the empirical evidence from $\mathrm{RCTs}^{357}$ for the presence of a personal element of response in adults ${ }^{358}$ with MDD by comparing the outcome variance in the an- ${ }^{359}$ tidepressant group with the outcome variance in the placebo ${ }^{360}$ group. Analyzing this variability ratio in 222 placebo- ${ }^{361}$ controlled RCTs from the last 40 years, we found no evi- ${ }^{362}$ dence for a larger variance in the antidepressant group than ${ }^{363}$ in the placebo group overall, or for any antidepressant alone. ${ }^{364}$ Thus, we found no empirical support for a treatment-by- ${ }^{365}$ patient interaction of antidepressants for the treatment of ${ }^{366}$ adults with MDD in RCTs. Further, we illustrated that infer- ${ }^{367}$ ences made on the basis of the observed outcomes in $\mathrm{RCTs}^{368}$ can be misleading and can encourage the assumption of in ${ }^{-369}$ dividual differences in the response to antidepressants.

Translating the population average effect of an intervention ${ }_{372}$ into an effect for the individual patient requires additional $\mathrm{l}_{373}$ assumptions when based on an RCT; one is that of a con-374 stant effect, that would make the results relevant to every $y_{375}$ patient [43]. One consequence of a constant effect is that $t_{376}$ the treatment does not affect the outcome variance, which $_{377}$ would therefore be expected to be equal in the treatment and ${ }_{378}$ placebo groups [33]. Studying the variance in the treatment ${ }_{379}$ and placebo groups of an RCT thus provides the means for ${ }_{380}$ quantifying differences in variability that may arise due $t_{381}$ the investigated treatment $[34,35]$. This approach allows inferences about a potential treatment-by-patient interaction. .822 Then, an increased variability in the antidepressant group ${ }_{383}$ compared with the placebo group, could arise due to individ-384 ual differences in response to the treatment [33]. An alter-385 native explanation would be the presence of two subgroup\$386 [33]: one subgroup of patients having a small or even neg-387 ative effect of treatment with antidepressants, another sub-388 group having a large effect of the treatment. Such a sce-389 nario would call for a stratified research design to identify the subgroup most likely to benefit from the treatment with antidepressants and the subgroup in which such a treatment would have no or even a negative effect, and for the subsequent practice of stratified medicine rather than personalized medicine. An analysis of the variability ratio does not allow for drawing conclusions on the individual level, and thus cannot distinguish between a potential treatment-by-patient interaction and that of a treatment-by-subgroup interaction. Yet, our results showing that the variance in the antidepressant group did not differ from the placebo group, may indicate that neither of those scenarios are likely.

Careful appreciation of the variance components of trials that are discernable by analyzing RCTs is necessary to recognize which conclusions can be derived from them. While an RCT can ideally provide an unbiased estimate of the effect of treatment by comparing the outcomes of the patients in the treatment group to the outcomes of the patients in the placebo group, it cannot inform on variation in effects between the patients [44]. Without knowledge about how the same patient would have fared under placebo assumptions about individual differences in the response to antidepressants are unfounded. Therefore, the observed outcomes of the treatment group in an RCT alone cannot provide any evidence for a potential treatment-by-patient interaction; rather, focusing on the differences in the observed outcomes of those receiving treatment can lead to the potentially misleading interpretation that some patients benefit more from the treatment than others, by ascribing all or much of the variation in their observed outcomes to characteristics of the individual. The labelling of patients in RCTs as "responders" or "non-responders", apart from the problems with dichotomizing continuous outcomes [45], is problematic. It alludes to the existence of an individual response to treatment that cannot be inferred from RCTs to begin with [30]. The often held viewpoint that the average treatment effect constitutes a simplified summary estimate of a range of responses to the treatment $[21,22,46]$ neglects the fact that RCTs cannot inform on the variation within and between patients (Senn, 2016a). Alternative to the often held assumption that the individual response to a treatment in an RCT is a trait of the individual, the response may rather, and perhaps most likely, not be a permanent characteristic but vary from occasion to occasion [30].

Direct evidence of a treatment-by-patient interaction can be provided by repeated crossover trials, including N-of- 1 trials. Yet, such trial designs are not without methodological challenges and are often impossible to conduct in conditions such as MDD; depressive symptoms often fluctuate, treatment effects may appear only after weeks of treatment, carry-over effects may arise if long-term changes to brain neurochemistry persist [47], and withdrawal effects may be 
prolonged [48]. However difficult, without the efforts to un-435 dertake these designs and analyses no clear understanding of whether a treatment-by-patient interaction is present can be ${ }_{436}$ reached. Given our finding of lacking empirical support for a treatment-by-patient interaction to antidepressants, there may be little reason to assume that some patients treated with antidepressants for depression will have a larger effect than the average effect demonstrated in RCTs. Thus, the promise for personalized medicine in antidepressant treatment of de- ${ }^{43}$ pression may be unfounded.

\section{Limitations}

There are limitations to our meta-analysis. First, we relied ${ }^{442}$ on previously extracted data from individual studies [10] and $^{443}$ did not extract the data ourselves. Second, the trials were ${ }^{444}$ overall of short duration and antidepressant trials generally apply strict inclusion criteria; it is possible that individual treatment effects could be observed with longer trial duration and in populations that differ from those usually included in clinical trials. Third, the HAMD is psychometrically flawed [49] and the variability ratio could potentially differ when analyzing other outcomes. Fourth, our meta-analysis using the CVR effect size statistic indicated lower variability in the antidepressant group compared with placebo. This finding differed from our finding for the VR. However, both methods have rationale $[33,34]$ and none of the analyses were indicative of a treatment-by-patient interaction. Fifth, while an increase in the variance in the antidepressant group compared with placebo would indicate a treatment-by-patient interaction, the reverse is not true - equal variance in the antidepressant and the placebo group does not eliminate the possibility of a treatment-by-patient interaction. Nevertheless, the simplest explanation for equal variances in the antidepressant and placebo group is that of a constant effect [33].

\section{Conclusions}

In our analysis of the variability ratio in RCTs we did not find any evidence for individual differences in treatment effects of antidepressants for depression in adults. Given that RCTs cannot provide direct evidence for individual treatment effects, our findings suggest that it may be most reasonable to assume that the average effect of antidepressants compared with placebo for depression applies to the individual patient.

\section{Conflicts of interest}

The authors report no conflicts of interest.

\section{Funding}

No funding was obtained for the study.

\section{Data sharing}

All data and code are available online (https://osf.io/5gpe4/).

\section{Acknowledgements}

We thank Cipriani et al. [10] for making their data available online.

\section{Contributions}

$\mathrm{KM}$, SW and PH conceived the study. KM conducted the analyses. KM wrote the first draft of the manuscript. All authors critically revised the manuscript for important intellectual content. 


\section{References}

Malhi GS, Mann JJ. Depression. The Lancet 2018;392(10161):2299-312.

2. WHO. World health organisation. Media centre. Depres $-{ }^{498}$ sion: Fact sheet. [Internet]. Vol. 2018. 2017. ${ }^{499}$ Available from: http://www.who.int/mediacentre ${ }^{500}$ factsheets/fs369/en/

3. Bauer M, Severus E, Moller HJ, Young AH, Disor- ${ }^{502}$ ders WTF on UD. Pharmacological treatment of ${ }^{503}$ unipolar depressive disorders: Summary of $\mathrm{wfsbp}^{504}$ guidelines. Int J Psychiatry Clin Pract [Internet]. ${ }^{505}$ 2017;21(3):166-76. Available from: https://www. ${ }^{506}$ ncbi.nlm.nih.gov/pubmed/28367707

4. Cleare A, Pariante CM, Young AH, Anderson IM, Christ- ${ }^{508}$ mas D, Cowen PJ, et al. Evidence-based guide- ${ }^{509}$ lines for treating depressive disorders with antide- ${ }^{510}$ pressants: A revision of the 2008 british association ${ }^{511}$ for psychopharmacology guidelines. J Psychophar- ${ }^{512}$ macol [Internet]. 2015;29(5):459-525. Avail- ${ }^{513}$ able from: https://www.ncbi.nlm.nih.gov/pubmed/ ${ }^{514}$ 25969470

5. Gelenberg AJ, Freeman MP, Markowitz JC, Rosenbaum ${ }^{516}$ JF, Thase M, Trivedi MH, et al. Practice guideline $e^{517}$ for the treatment of patients with major depressive ${ }^{518}$ disorder. American Psychiatric Association; 2010. ${ }^{519}$

6. Malhi GS, Bassett D, Boyce P, Bryant R, Fitzgerald PB, ${ }_{521}^{520}$ Fritz K, et al. Royal australian and new zealand ${ }_{522}$ college of psychiatrists clinical practice guidelines for mood disorders. Aust N Z J Psychiatry [In-523 ternet]. 2015;49(12):1087-206. Available from:524 https://www.ncbi.nlm.nih.gov/pubmed/26643054 525

7. NICE. Depression in adults: Recognition and manage- ${ }_{527}^{526}$ ment. Clinical guideline [cg90]. [Internet]. 2009 ${ }_{528}^{527}$ Available from: https://www.nice.org.uk/guidance/ $\operatorname{cg} 90$

8. Kennedy SH, Lam RW, McIntyre RS, Tourjman SV, ${ }_{531}^{530}$ Bhat V, Blier P, et al. Canadian network for ${ }_{532}$ mood and anxiety treatments (canmat) 2016 clin- $^{532}$ ical guidelines for the management of adults with ${ }_{534}^{533}$ major depressive disorder: Section 3. Pharma- ${ }_{535}$ cological treatments. Can J Psychiatry [Internet] ${ }_{536}^{535}$ 2016;61(9):540-60. Available from: https://www. ncbi.nlm.nih.gov/pubmed/27486148

537

9. Arroll B, Elley CR, Fishman T, Goodyear-Smith ${ }_{539}^{538}$ FA, Kenealy T, Blashki G, et al. Antidepres- ${ }^{539}$ sants versus placebo for depression in primary ${ }^{540}$ care. Cochrane Database Syst Rev [Internet]. ${ }^{541}$ 2009;(3):CD007954. Available from: https://www. ${ }^{542}$ ncbi.nlm.nih.gov/pubmed/19588448
10. Cipriani A, Furukawa TA, Salanti G, Chaimani A, Atkinson LZ, Ogawa Y, et al. Comparative efficacy and acceptability of 21 antidepressant drugs for the acute treatment of adults with major depressive disorder: A systematic review and network meta-analysis. Lancet [Internet]. 2018;391(10128):1357-66. Available from: https: //www.ncbi.nlm.nih.gov/pubmed/29477251

11. Fournier JC, DeRubeis RJ, Hollon SD, Dimidjian S, Amsterdam JD, Shelton RC, et al. Antidepressant drug effects and depression severity: A patient-level meta-analysis. JAMA [Internet]. 2010;303(1):4753. Available from: https://www.ncbi.nlm.nih.gov/ pubmed/20051569

12. Gibbons RD, Hur K, Brown $\mathrm{CH}$, Davis JM, Mann JJ. Benefits from antidepressants: Synthesis of 6-week patient-level outcomes from double-blind placebo-controlled randomized trials of fluoxetine and venlafaxine. Arch Gen Psychiatry [Internet]. 2012;69(6):572-9. Available from: https://www. ncbi.nlm.nih.gov/pubmed/22393205

13. Jakobsen JC, Katakam KK, Schou A, Hellmuth SG, Stallknecht SE, Leth-Moller K, et al. Selective serotonin reuptake inhibitors versus placebo in patients with major depressive disorder. A systematic review with meta-analysis and trial sequential analysis. BMC Psychiatry [Internet]. 2017;17(1):58. Available from: https://www.ncbi. nlm.nih.gov/pubmed/28178949

14. Kirsch I, Deacon BJ, Huedo-Medina TB, Scoboria A, Moore TJ, Johnson BT. Initial severity and antidepressant benefits: A meta-analysis of data submitted to the food and drug administration. PLoS Med [Internet]. 2008;5(2):e45. Available from: https: //www.ncbi.nlm.nih.gov/pubmed/18303940

15. NICE. National institute for health and care excellence. Depression: Management of depression in primary and secondary care. Clinical practice guideline number 23. [Internet]. Vol. 2018. National Institute for Health; Clinical Excellence; 2004. Available from: http://webarchive.nationalarchives. gov.uk/20080612001911/https://www.nice.org.uk/ guidance/index.jsp?action=download \&o $=29617$

16. Turner EH, Matthews AM, Linardatos E, Tell RA, Rosenthal R. Selective publication of antidepressant trials and its influence on apparent efficacy. $\mathrm{N}$ Engl J Med [Internet]. 2008;358(3):252-60. Available from: https://www.ncbi.nlm.nih.gov/pubmed/ 18199864

17. Munkholm K, Paludan-Muller AS, Boesen K. Consider- 
ing the methodological limitations in the evidence 593 base of antidepressants for depression: A reanal-594 ysis of a network meta-analysis. BMJ Open [In-595 ternet]. 2019;9(6):e024886. Available from: https:596 //www.ncbi.nlm.nih.gov/pubmed/31248914

597

18. Hamilton M. Development of a rating scale for pri-598 mary depressive illness. Br J Soc Clin Psychol [In-599 ternet]. 1967;6(4):278-96. Available from: https:600 //www.ncbi.nlm.nih.gov/pubmed/6080235 601

19. Ioannidis JP. Effectiveness of antidepressants: An evi- ${ }_{603}^{602}$ dence myth constructed from a thousand random- ${ }_{604}$ ized trials? Philos Ethics Humanit Med [Internet]. 2008;3:14. Available from: https://www.ncbi.nlm.605 nih.gov/pubmed/18505564

20. Carter GC, Cantrell RA, Victoria Z, Haynes VS, ${ }_{608}^{607}$ Phillips G, Alatorre CI, et al. Comprehensive review of factors implicated in the heterogeneity of response in depression. Depress Anxiety [Inter-610 net]. 2012;29(4):340-54. Available from: https:611 //www.ncbi.nlm.nih.gov/pubmed/22511365 612

21. Cipriani A, Salanti G, Furukawa TA, Egger $M_{, 614}^{613}$ Leucht S, Ruhe HG, et al. Antidepressants might work for people with major depression: Where $\mathrm{do}^{615}$ we go from here? Lancet Psychiatry [Internet]. ${ }^{616}$ 2018;5(6):461-3. Available from: https://www. ${ }^{617}$ ncbi.nlm.nih.gov/pubmed/29628364

22. Holsboer F. How can we realize the promise of per- ${ }^{619}$ sonalized antidepressant medicines? Nat Rev Neu- ${ }^{620}$ rosci [Internet]. 2008;9(8):638-46. Available from: ${ }^{.21}$ https://www.ncbi.nlm.nih.gov/pubmed/18628772 ${ }^{622}$

23. Boland JR, Duffy B, Myer NM. Clinical utility of ${ }^{623}$ pharmacogenetics-guided treatment of depression ${ }^{624}$ and anxiety. Personalized Medicine in Psychiatry. ${ }_{626}^{625}$ 2018;7-8:7-13.

24. Carrillo-Roa T, Labermaier C, Weber P, Herzog ${ }^{627}$ DP, Lareau C, Santarelli S, et al. Common ${ }^{628}$ genes associated with antidepressant response in ${ }^{629}$ mouse and man identify key role of glucocorti- ${ }^{630}$ coid receptor sensitivity. PLoS Biol [Internet]. ${ }^{631}$ 2017;15(12):e2002690. Available from: https:// ${ }^{632}$ www.ncbi.nlm.nih.gov/pubmed/29283992

25. Cattaneo A, Ferrari C, Uher R, Bocchio-Chiavetto ${ }^{634}$ L, Riva MA, Consortium MRCI, et al. Abso- ${ }^{635}$ lute measurements of macrophage migration in $^{636}$ hibitory factor and interleukin-1-beta mRNA levels ${ }^{637}$ accurately predict treatment response in depressed 638 patients. Int J Neuropsychopharmacol [Internet].6з9 2016;19(10). Available from: https://www.ncbi.640 nlm.nih.gov/pubmed/27207917
26. Jha MK, Trivedi MH. Personalized antidepressant selection and pathway to novel treatments: Clinical utility of targeting inflammation. Int J Mol Sci [Internet]. 2018;19(1). Available from: https://www. ncbi.nlm.nih.gov/pubmed/29329256

27. Green E, Goldstein-Piekarski AN, Schatzberg AF, Rush AJ, Ma J, Williams L. Personalizing antidepressant choice by sex, body mass index, and symptom profile: An iSPOT-d report. Personalized Medicine in Psychiatry [Internet]. 2017;1-2:65-73. Available from: http://www.sciencedirect.com/science/ article/pii/S2468171716300242

28. Leuchter AF, Cook IA, Hunter AM, Korb AS. A new paradigm for the prediction of antidepressant treatment response. Dialogues Clin Neurosci [Internet]. 2009;11(4):435-46. Available from: https: //www.ncbi.nlm.nih.gov/pubmed/20135901

29. Schuyler D. Trial and error [Internet]. Vol. 2019. Los Angeles Times; 2009. Available from: https://www.latimes.com/archives/ la-xpm-2009-aug-03-he-depression-drug-choice3-story. html

30. Senn S. Statistical pitfalls of personalized medicine. Nature [Internet]. 2018;563(7733):619-21. Available from: https://www.ncbi.nlm.nih.gov/pubmed/ 30482931

31. Senn S. Individual response to treatment: Is it a valid assumption? BMJ [Internet]. 2004;329(7472):9668. Available from: https://www.ncbi.nlm.nih.gov/ pubmed/15499115

32. Senn S. Mastering variation: Variance components and personalised medicine. Stat Med [Internet]. 2016;35(7):966-77. Available from: https://www. ncbi.nlm.nih.gov/pubmed/26415869

33. Cortes J, Gonzalez JA, Medina MN, Vogler M, Vilaro M, Elmore $\mathrm{M}$, et al. Does evidence support the high expectations placed in precision medicine? A bibliographic review. F1000Res [Internet]. 2018;7:30. Available from: https://www. ncbi.nlm.nih.gov/pubmed/31143439

34. Nakagawa S, Poulin R, Mengersen K, Reinhold K, Engqvist L, Lagisz M, et al. Meta-analysis of variation: Ecological and evolutionary applications and beyond. Methods in Ecology and Evolution. 2015;6(2):143-52.

35. Winkelbeiner S, Leucht S, Kane JM, Homan P. Evaluation of differences in individual treatment response in schizophrenia spectrum disorders: A metaanalysis. JAMA Psychiatry [Internet]. 2019; Avail- 
able from: https://www.ncbi.nlm.nih.gov/pubmed/691 31158853

36. Schneider LS, Nelson JC, Clary CM, Newhouse ${ }^{693}$ P, Krishnan KR, Shiovitz T, et al. An 8-week ${ }^{694}$ multicenter, parallel-group, double-blind, placebo- $^{695}$ controlled study of sertraline in elderly outpatients ${ }^{696}$ with major depression. Am J Psychiatry [Inter-697 net]. 2003;160(7):1277-85. Available from: https:698 //www.ncbi.nlm.nih.gov/pubmed/12832242 699

37. Nierenberg AA, DeCecco LM. Definitions of antidepres- ${ }^{700}$ sant treatment response, remission, nonresponse,701 partial response, and other relevant outcomes: $\mathrm{A}_{702}$ focus on treatment-resistant depression. J Clin Psy-703 chiatry [Internet]. 2001;62 Suppl 16:5-9. Avail-704 able from: https://www.ncbi.nlm.nih.gov/pubmed/705 11480882

38. Montgomery SA, Asberg M. A new depression scale ${ }_{707}$ designed to be sensitive to change. British Journal ${ }_{708}$ of Psychiatry [Internet]. 1979;134(4):382-9.709 Available from: http://www.scopus.com/inward/ $/ 710$ record.url?eid=2-s2.0-0018425438\&partnerID= 40\&md5=c09a143849d57a8b804002ee802c72e 7

39. Hedges LV, Nowell A. Sex differences in mental test scores, variability, and numbers of high-scoring individuals. Science [Internet]. 1995;269(5220):415. Available from: https://www.ncbi.nlm.nih.gov/ pubmed/7604277

40. Viechtbauer W. Conducting meta-analyses inRwith themetaforPackage. Journal of Statistical Software. 2010;36(3).

41. Schwarzer G. Meta: An r package for meta-analysis. $R$ News. 2007;7(3):40-5.

42. Dube S, Dellva MA, Jones M, Kielbasa W, Padich R, Saha A, et al. A study of the effects of ly2216684, a selective norepinephrine reuptake inhibitor, in the treatment of major depression. J Psychiatr Res [Internet]. 2010;44(6):356-63. Available from: https: //www.ncbi.nlm.nih.gov/pubmed/19909980

43. Holland PW. Statistics and causal inference. Journal of the American Statistical Association [Internet]. 1986;81(396):945-60. Available from: https://www.tandfonline.com/doi/abs/ 10.1080/01621459.1986.10478354

44. Senn S. Individual therapy: New dawn or false dawn? Drug Information Journal. 2001;35(4):1479-94.

45. Altman DG, Royston P. The cost of dichotomising continuous variables. BMJ [Internet]. 2006;332(7549):1080. Available from: https://www.ncbi.nlm.nih.gov/pubmed/16675816
46. Herzog DP, Beckmann H, Lieb K, Ryu S, Muller MB. Understanding and predicting antidepressant response: Using animal models to move toward precision psychiatry. Front Psychiatry [Internet]. 2018;9:512. Available from: https://www.ncbi.nlm. nih.gov/pubmed/30405454

47. Senn S, Lambrou D. Robust and realistic approaches to carry-over. Stat Med [Internet]. 1998;17(24):284964. Available from: https://www.ncbi.nlm.nih.gov/ pubmed/9921606

48. Davies J, Read J. A systematic review into the incidence, severity and duration of antidepressant withdrawal effects: Are guidelines evidence-based? Addict Behav [Internet]. 2019;97:111-21. Available from: https://www.ncbi.nlm.nih.gov/pubmed/30292574

49. Bagby RM, Ryder AG, Schuller DR, Marshall MB. The hamilton depression rating scale: Has the gold standard become a lead weight? Am J Psychiatry [Internet]. 2004;161(12):2163-77. Available from: https://www.ncbi.nlm.nih.gov/pubmed/15569884 